\title{
Hochrisikopatient|-innen für VTE profitieren von zusätzlicher postoperativer IPK
}

\author{
Lobastov K et al. Intermittent Pneumatic Compression in Addition to Standard Prophylaxis of Postoperative Venous Thromboembolism in Extremely High-risk \\ Patients (IPC SUPER): A Randomized Controlled Trial. Ann Surg 2021; 274: 63-69 doi:10.1097/SLA.0000000000004556
}

Ein signifikanter Risikofaktor für venösen Thromboembolismus (VTE) sind große chirurgische Eingriffe. Bei Patient/-innen mit extrem hohem Risiko ist die postoperative Standardprophylaxe unzureichend. Lobastov et al. gingen der Frage nach, ob die zusätzliche intermittierende pneumatische Kompression (IPK) das Risiko für postoperative VTE reduziert.

Der Caprini-Score gilt als das am besten validierte Prognosemodell zur individuellen Risikoeinschätzung. Er zeigte weltweit bei über 3 Millionen chirurgischen Patient/-innen eine starke Korrelation mit der Häufigkeit symptomatischer VTE-Ereignisse. Ein solches trat bei einem Score $\geq 11$ trotz Standardprophylaxe in 59\% der Fälle auf.

Die Studie wurde an 2 Standorten in Moskau, Russische Föderation, zwischen Februar 2017 und Oktober 2018 durchgeführt. Aufgenommen wurden 407 Patient/-innen im Alter von $\geq 40$ Jahren, bei denen ein großer chirurgischer Eingriff durchgeführt werden sollte und die einen Caprini-Score $\geq 11$ aufwiesen. Sie wurden randomisiert für die alleinige postoperative VTE-Standardprophylaxe $(n=203)$ und für zusätzliche IPK-Therapie ( $n=204)$. Beide Gruppen waren nach den wesentli- chen demografischen und klinischen Charakteristiken vergleichbar und nach dem Caprini-Score gut ausgewogen. Die Standardprophylaxe umfasste Kompressionsstrümpfe der Klasse 1 mit einem Druck von 18-21 mmHg am Knöchel und die Gabe von niedermolekularem Heparin (LMWH). Die Kompressionsstrümpfe wurden präoperativ angelegt und während des gesamten stationären Aufenthaltes getragen. Die IPK wurde im Operationssaal vor der Inzision begonnen oder auf der Intensivstation innerhalb von 12 Stunden postoperativ. Die Behandlung erfolgte während des gesamten Aufenthaltes in der chirurgischen Abteilung über 18 Stunden täglich. Primärer Outcome war eine asymptomatische, mit Duplex-Sonografie entdeckte VTE der Beine. Duplex-Sonografien wurden präoperativ durchgeführt und nach dem Eingriff alle 3-5 Tage bis zur Entlassung oder bis zum Tod.

Das Follow-up während des stationären Aufenthaltes war bei allen Patient/-innen möglich. Der primäre Outcome ereignete sich bei einem Patienten/einer Patientin $(0,5 \%)$ in der IPK-Gruppe und bei $34 \mathrm{~Pa}$ tient/-innen $(16,7 \%)$ in der Kontrollgruppe. Das relative Risiko (RR) betrug 0,03 (95\%-KI 0,01-0,21). In der IPK-Gruppe traten keine Lungenembolien auf, in der Kontrollgruppe mit alleiniger Standard- prophylaxe waren es 5 (2,5\%) Fälle (RR 0,09; 95\%-KI 0,01-1,63). Postoperative Todesfälle gab es $6(2,9 \%)$ in der IPK-Gruppe und $10(4,9 \%)$ in der Kontrollgruppe (RR 0,50; 95\%-KI 0,50-1,60). Ein Followup nach 30 Tagen postoperativ war bei 193 Patient/-innen in der IPK-Gruppe und bei 194 in der Kontrollgruppe möglich. Nach 180 Tagen waren es entsprechend 167 und 158. Zu beiden Terminen wurden keine neu aufgetretenen VTE diagnostiziert. Die Mortalität beider Gruppen unterschied sich nicht.

FAZIT

Laut den Autor/-innen führte die zusätzliche Therapie mit IPK bei chirurgischen Patient/-innen mit sehr hohem Risiko für VTE zu einer signifikant niedrigeren Inzidenz asymptomatischer VTE. Die Anwendung des Caprini-Scores ermögliche die $\mathrm{Pa}$ tient/-innen zu identifizieren, die von der kombinierten VTE-Prophylaxe am meisten profitieren.

Gabriele Dobler, Berlin 